\title{
From DEM Simulations towards a Continuum Theory of Granular Matter
}

\author{
Stefan Luding \\ Institute for Computer Applications 1, Pfaffenwaldring 27, 70569 Stuttgart, Germany \\ e'-mail: lui@ica1.uni-stuttgart.de - URL: http://www.ica1.uni-stuttgart.de/ /ui
}

ABSTRACT: One essential question in material sciences is how to bridge the gap between the microscopic picture and a macroscopic description. The former involves contact forces and deformations, whereas the latter concerns tensorial quantities like the stress or the velocity gradient.

A two-dimensional shear-cell filled with disks of different sizes is examined by means of a "microscopic" discrete element method (DEM). Applying a consistent averaging formalism, one can obtain scalar- and vectorJfields as well as classical tensorial macroscopic quantities like fabric, stress or velocity gradient and, in addition, Emicropolar quantities like curvature or couple-stress.

\section{INTRODUCTION}

The macroscopic balance equations for mass, momentum and energy can be used for the modeling of the behavior of granular media. However, they rely on constitutive relations between the physical quantities, in order to close the system of equations. The determination of both the physical quantities, important for the behavior of the system, and their inter-relations are subject of current research (Behringer and Jenkins 1997; Herrmann et al. 1998; Vermeer et al. 2000). TOne possible way to obtain an observable like, for example, the stress is to perform experiments with 'photo-elastic material (Veje et al. 1999; Howell and Behringer 1999; Howell et al. 1999) which allows for non-invasive force measurements. The alternative is' to perform discrete particle simulations (Cundall and Strack 1979; Herrmann et al. 1998; Lätzel et al. $2000 \mathrm{~b}$ and to average over the "microscopic" forces and contact vectors in the simulation. A typcal snap-shot from a two-dimensional DEM simulation is displayed in Fig. 1.

Besides the trivial definitions for averages over scalar and vectorial quantities like density, velocity, and particle-spin, one can find differing definitions for stress and strain averaging procedures in the literature (Bathurst and Rothenburg 1988; Kruyt and Rothenburg 1996; Liao et al. 1997; Calvetti et al. 1997; Lätzel et al. 2000b; Dedecker et al. 2000).

The aim of this paper is to review recent results for tensorial, averaged continuum quantities involving also micro-polar aspects (Lätzel et al. 2000a; Zervos et al. 2000).

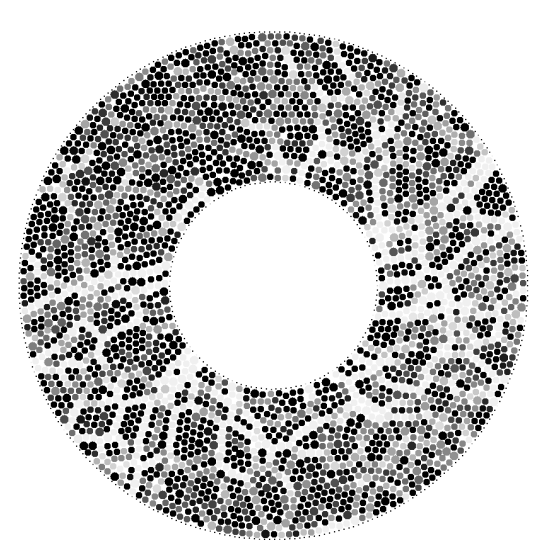

Figure 1: Representative snapshot of the model system. Light and dark grey indicate large and small values of the contact potential energy, respectively.

In section 2 our averaging method is introduced and applied to obtain some macroscopic fields in section 3 . Section 4 contains the definitions and averaging strategies for fabric, stress, and elastic deformation gradient. The particle rotations are taken into account in section 5, averages are presented for selected macroscopic quantities in section 6 and material parameters are discussed in section 7 .

\section{FROM MICRO- TO MACRO-DESCRIPTION}

In a microscopic, discrete picture, the knowledge of the forces acting on each particle is sufficient to model the dynamics and the statics of the system. Tensorial quantities like the stress $\sigma$ or the strain $\varepsilon$ are not re- 
quired for a discrete model. In order to establish a correspondence to continuum theories one has to compute tensorial fields as well as scalar material properties like, e. g., the bulk and shear moduli (Cambou et al. 1995; Kruyt and Rothenburg 1996; Emeriault and Chang 1997; Liao et al. 1997; Lätzel et al. 2000b; Luding et al. 2000; Lätzel et al. 2000a). In the following, a consistent averaging strategy is introduced.

\subsection{Averaging Strategy}

Most of the measurable quantities in granular materials vary strongly both in time and on short distances. At a fixed point in space, one has either a particle or one finds an empty space. Therefore, density flips between the two values zero and $\varrho^{p}$, where the latter is the material density. During the "homogenization" presented in the next subsection, we average over space and time, in order to reduce the fluctuations. The averaging over space is an option in systems with some translational or rotational invariance (simple shear or ring shear apparatus); averaging over long times is feasible only in a steady state situation. In a ring shear apparatus, as introduced later in section 6, all points at a certain distance from the origin are equivalent to each other and, after several shear cycles, the system reaches a quasi-steady state. Averaging over many snapshots is somehow equivalent to an ensemble average. However, we remark that different snapshots are not necessarily independent of each other (Lätzel et al. 2000b) and the duration of a simulation might be too short to explore a representative part of the phase space.

The final assumption for the averaging procedure presented below, is that all quantities are smeared out over one particle. This is a good approximation for the density, but not for the stress. However, since we aim at macroscopic, averaged quantities, it is not our goal to solve for the stress field inside one particle. Details of the position dependency inside the particles are presumably smeared out due to the averaging in space and time.

\subsection{Averaging Formalism}

The mean value of some quantity $Q$ is defined as

$$
Q=\frac{1}{V} \sum_{p \in V} w_{V}^{p} V^{p} Q^{p}
$$

with the averaging volume $V$ and the particle volume $V^{p}$. $Q^{p}=\sum_{c=1}^{C^{p}} Q^{c}$ is a quantity attributed to particle $p$, where the quantity $Q^{c}$ is attributed to contact $c$ of particle $p$ with $C^{p}$ contacts. The weight $w_{V}^{p}$ accounts for the particle's contribution to the average, and corresponds to the fraction of the particle volume that is covered by the averaging volume. Since an exact calculation of the area of a circular particle that lies in an arbitrary volume is rather complicated, we assume that the boundaries of $V$ are locally straight, i.e. we cut the particle in slices, see (Lätzel et al. 2000b) for details. In the following section possible choices for observables are summarized and discussed.

\section{MACROSCOPIC FIELDS}

In the following, the averaging formalism in Eq. (11) is applied to obtain various macroscopic quantities. For example, the quantity $Q^{p}$ can be chosen as $\varrho^{p}$ in order to obtain the density, as $\varrho^{p} \boldsymbol{v}^{p}$ in order to obtain the momentum density, or as $(1 / 2) \varrho^{p}\left(\boldsymbol{v}^{p}\right)^{2}$ for the kinetic energy density. In table 11, the macroscopic fields as well as particle-attributed quantities are collected. In this study, two tensors with no symbol in between mean a dyadic product, whereas '.' is used for the scalar product, i. e. the order-reduction by one for each of the two tensors at left and right.

Table 1: Macroscopic fields computed using the averaging formalism in Eq. (11) using particle properties

\begin{tabular}{|l|c|}
\hline macroscopic quantity & $Q^{p}$ \\
\hline \hline volume fraction $\nu$ & 1 \\
density $\varrho$ & $\varrho^{p}$ \\
flux density $\nu \boldsymbol{v}$ & $\boldsymbol{v}^{p}$ \\
momentum density $\varrho \boldsymbol{v}$ & $\varrho^{p} \boldsymbol{v}^{p}$ \\
kinetic energy density $\frac{1}{2} \varrho \boldsymbol{v}^{2}$ & $\frac{1}{2} \varrho^{p}\left(\boldsymbol{v}^{p}\right)^{2}$ \\
dynamic stress tensor ${ }^{d} \boldsymbol{\sigma}$ & $\varrho^{p} \boldsymbol{v}^{p} \boldsymbol{v}^{p}$ \\
\hline
\end{tabular}

\subsection{Volume Fraction}

As a first example for an averaged scalar quantity, the local volume fraction $\nu$, see Tab. 1, is directly related to the local density via $\varrho \approx \varrho^{p} \nu$.

\subsection{Flux Density}

As a tensorial quantity of first order (vector), the velocity field $\boldsymbol{v}$ is obtained when dividing the flux density $\nu \boldsymbol{v}$ by the volume fraction $\nu$. The momentum density is thus $\varrho \boldsymbol{v} \approx \varrho^{p} \nu \boldsymbol{v}$.

\subsection{Velocity Gradient}

Given the velocity field, it is possible to compute the velocity gradient $\boldsymbol{\nabla} \boldsymbol{v}$, a tensor of second order, by means of numerical differentiation. With $\boldsymbol{\nabla} \boldsymbol{v}$ one has the components of the symmetric deformation rate

$$
D_{\alpha \beta}=\frac{1}{2}\left[\frac{\partial v_{\alpha}}{\partial \beta}+\frac{\partial v_{\beta}}{\partial \alpha}\right]
$$

and the anti-symmetric continuum rotation rate

$$
W_{\alpha \beta}=\frac{1}{2}\left[\frac{\partial v_{\alpha}}{\partial \beta}-\frac{\partial v_{\beta}}{\partial \alpha}\right]
$$


with the coordinates $\alpha$ and $\beta$. In the special case of cylindrical symmetry with radial direction $r$ and angular direction $\phi$, Eqs. (2) and (3) reduce to

$$
D_{r \phi}=\frac{1}{2}\left[\frac{\partial v_{\phi}}{\partial r}-\frac{v_{\phi}}{r}\right]
$$

and

$$
W_{r \phi}=\frac{1}{2}\left[\frac{\partial v_{\phi}}{\partial r}+\frac{v_{\phi}}{r}\right],
$$

see (Luding et al. 2000) for details and (Zervos et al. 2000) for a similar approach.

\subsection{Energy Density}

The kinetic energy density is only a function of the velocity of the particles in the averaging volume and it is related to the trace of the dynamic stress tensor. However, we do not discuss this quantity here, since the system examined later is quasi-static so that the dynamic contributions are of minor importance (Luding et al. 2000; Lätzel et al. 2000a).

\section{MACROSCOPIC TENSORIAL QUANTITIES}

In this section, the averaged, macroscopic tensorial quantities in our model system are introduced. The fabric tensor describes the statistics of the contact directions, the stress tensor describes the stress distribution due to the contact forces, and the elastic deformation gradient is a measure for the elastic, reversible deformations due to the stress.

Table 2: Macroscopic tensorial quantities computed with Eq. (11) using contact-attributed properties preaveraged over single particles

\begin{tabular}{|l|c|}
\hline macroscopic quantity & $Q^{p}$ \\
\hline \hline fabric tensor $\mathbf{F}$ & $\sum_{c=1}^{C^{p}} \boldsymbol{n}^{c} \boldsymbol{n}^{c}$ \\
static stress tensor $\boldsymbol{\sigma}$ & $\frac{1}{V^{p}} \sum_{c=1}^{C^{p}} \boldsymbol{f}^{c} \boldsymbol{l}^{p c}$ \\
deformation gradient $\boldsymbol{\epsilon}$ & $\frac{\pi h}{V^{p}} \sum_{c=1}^{C^{p}} \boldsymbol{\Delta}^{p c} \boldsymbol{l}^{p c} \cdot \mathbf{F}^{-1}$ \\
\hline
\end{tabular}

\subsection{Fabric Tensor}

In assemblies of grains, the forces are transmitted from one particle to the next only at the contacts of the particles. Therefore, the local geometry and direction of each contact is important (Lätzel et al. 2000b). The fabric tensor, in our definition, involves the contact normal vector $\boldsymbol{n}^{c}$, related to the so-called branch vector via $\boldsymbol{l}^{p c}=a_{p} \boldsymbol{n}^{c}$, with particle radius $a_{p}$. For each contact the dyadic product $\boldsymbol{n}^{c} \boldsymbol{n}^{c}$ is used, i. e. a degenerate tensor of order two with non-zero value in $\boldsymbol{n}^{c}$-direction only. In average over many contacts (and over many particles) one obtains the fabric tensor.

The fabric tensor in table 2 is symmetric by definition and thus consists of up to three independent scalar quantities in two dimensions. The first of them, the trace (or volumetric part) $F_{V}=\operatorname{tr}(\mathbf{F})=\left(F_{\max }+\right.$ $\left.F_{\min }\right)$, is the contact number density, with the major and the minor eigenvalues $F_{\max }$ and $F_{\min }$, respectively. With other words, one obtains the relation $\operatorname{tr}(\mathbf{F})=\nu C$ with reasonable accuracy for monodisperse particles, where $C$ is the average number of contacts per particle. For polydisperse size distribution functions, a multiplicative correction factor was recently proposed (Madadi et al. 2001), which depends on the first three moments of the size distribution function only.

The second scalar, the deviator $F_{D}=F_{\max }-F_{\min }$, accounts for the anisotropy of the contact network to first order, and the third, the angle $\phi_{F}$, gives the orientation of the "major eigenvector", i. e. the eigenvector corresponding to $F_{\max }$, defined with respect to the radial outwards direction for cylindrical symmetry. In other words, the contact probability distribution is proportional to the function $F_{V}+F_{D} \cos \left(2\left(\phi-\phi_{F}\right)\right)$ (Lätzel et al. 2000b), when averaged over many particles. Note that this approximation, a fourier expansion up to second order, is not always sufficient, i. e. a more general fabric tensor of rank four should be used (Mehrabadi et al. 1988; Herrmann et al. 1998).

\subsection{Stress Tensor}

The stress tensor $\boldsymbol{\sigma}$ is a measure for the force $\boldsymbol{f}=\boldsymbol{\sigma}$. $\boldsymbol{n}$, acting on an imaginary surface with normal $\boldsymbol{n}$, per unit area. A force is equivalent to some momentum transfer per unit time, so that also the momentum flux density contributes to the stress in a dynamic way. The dynamic stress, see table 11, corresponds to the stress in an ideal gas, whereas the static stress, see table 2 , is caused by the transfer of forces from a contact to the center of mass of a particle.

In order to account for both the stress and the distance of transfer, the static component of the stress tensor (Rothenburg and Selvadurai 1981; Satake and Jenkins 1988; Kruyt and Rothenburg 1996; Lätzel et al. 2000b) is defined as the dyadic product of the force $\boldsymbol{f}^{c}$ acting at contact $c$ with the corresponding branch vector, see table \&. Note that the formerly introduced surface quantity is here expressed as the sum over all particles in the averaging volume, with the respective weight factor in Eq. (11), for a detailed derivation see (Lätzel et al. 2000b).

\subsection{Elastic Deformation Gradient}

In order to reach the final goal, i.e. to measure the material properties of a granular ensemble, one is interested, e.g., in the stress-strain relationship of the material. The strain $\varepsilon$ can be obtained by time integra- 
tion of the velocity gradient, see subsection 3.2, and subsequent symmetrization and linearization. Here, an alternative quantity is introduced by application of "Voigt's hypothesis", i. e. assuming that the deformation is uniform and that every particle displacement conforms to the corresponding mean displacement field, but fluctuates about it (Liao et al. 1997; Lätzel et al. 2000b). The deformation gradient in table 2 is calculated for the special case of two-dimensional disks with height $h$.

This relates the actual deformations to a virtual, stress-free reference state where all contacts start to form, i.e. the particles are just touching, see (Lätzel et al. 2000b) for a detailed derivation. The result is a non-symmetric tensor $\epsilon$, which is not the strain. Instead, we refer to it as the elastic deformation gradient, since it accounts only for reversible (elastic) deformations.

\section{ROTATIONAL DEGREES OF FREEDOM}

Due to the particles' surface roughness, forces are transmitted also tangentially and thus granular particles will rotate. Therefore, also micro-polar macroscopic quantities related to the rotational degrees of freedom are of interest (Lätzel et al. 2000a; Zervos et al. 2000). The particle rotation, the couple-stress and the curvature are discussed in this section.

Table 3: Tensorial quantities, connected to the rotational degrees of freedom, computed with Eq. (1)

\begin{tabular}{|l|c|}
\hline macroscopic quantity & $Q^{p}$ \\
\hline \hline spin density $\nu \boldsymbol{\omega}$ & $\boldsymbol{\omega}^{p}$ \\
couple stress $\mathbf{M}$ & $\frac{1}{V^{p}} \sum_{c=1}^{C^{p}}\left(\boldsymbol{l}^{p c} \times \boldsymbol{f}^{c}\right) \boldsymbol{l}^{p c}$ \\
elastic curvature $\boldsymbol{\kappa}$ & $\frac{\pi h}{V^{p}} \sum_{c=1}^{C^{p}}\left(\boldsymbol{l}^{p c} \times \boldsymbol{\Delta}^{p c}\right) \boldsymbol{l}^{p c} \cdot \boldsymbol{F}^{-\mathbf{1}}$ \\
\hline
\end{tabular}

\subsection{Particle Rotation}

The mean angular velocity of the particles is obtained by using $Q^{p}=\boldsymbol{\omega}^{p}$, see table 3. Note that $\boldsymbol{\omega}$ also contains the continuum angular velocity

$$
\boldsymbol{\omega}_{c}=\mathbf{I} \times \mathbf{W},
$$

i. e. the vector product ' $x$ ' of the continuum rotation rate from Eq. (3) and the unit tensor I. The excess rotation, or particle eigen rotation, with respect to the underlying mean motion, is thus

$$
\boldsymbol{\omega}^{*}=\boldsymbol{\omega}-\boldsymbol{\omega}^{c},
$$

i. e. the total mean spin minus the continuum spin.

\subsection{Couple stress}

In the framework of a Cosserat continuum (Zervos et al. 2000) one has, in addition to the stress, also the couple stress $\mathbf{M}$. When an applied stress leads to a deformation, an applied couple-stress causes a rotational motion. It can thus be defined in analogy to the stress by replacing the force by the torque due to the tangential component of the force, see table 3. In a two dimensional system with cylindrical symmetry, only the components $M_{z r}$ and $M_{z \phi}$ of the tensor survive. Note that $\mathbf{M}=\mathbf{0}$, when the sum of the torques acting on spherical particles vanishes by definition in static equilibrium.

\subsection{Elastic Curvature}

In analogy to the elastic deformation gradient, we define the curvature $\kappa$ by replacing the overlap with the respective axial vector, $\boldsymbol{l}^{p c} \times \Delta^{p c}$, see table 3. This leads to a measure for the reversible or "frozen in" rotations in the system. Consequently, in static equilibrium of spherical particles, one has $\boldsymbol{\kappa}=\mathbf{0}$.

\section{RESULTS}

The elementary units of granular materials are mesoscopic grains. With DEM (Lätzel et al. 2000b; Lätzel et al. 2000a) the grains are treated as rigid particles but their local deformation at their contact points is realized as virtual overlaps. We relate the interaction forces to this overlap $\delta$ and to the tangential displacement of two particles during contact. The force laws used are material dependent and have to be validated by comparison with experimental measurements (Foerster et al. 1994; Labous et al. 1997; Falcon et al. 1998; Luding 1998).

\subsection{The Model System}

In the simulations presented in this study, a twodimensional Couette shear-cell is used. $N$ disks are confined between an outer ring and an inner ring with radius $R_{o}$ and $R_{i}$, respectively. The particles are of slightly different size $d_{\text {small }}=7.42 \mathrm{~mm}$ and $d_{\text {large }}=8.99 \mathrm{~mm}$, in order to reduce ordering effects. These boundary conditions are based on an experiment (Howell and Behringer 1999; Veje et al. 1999); for more details and other simulations, see (Lätzel et al. 2000b; Luding et al. 2000).

The outer wall is fixed and the inner wall rotates and thus introduces a slow shear deformation to the system. The simulations are started in a dilute state with an extended outer ring while the inner ring already rotates counter-clockwise with constant angular velocity $\Omega=2 \pi / T_{i}=0.1 \mathrm{~s}^{-1}$ and period $T_{i}=62.83 \mathrm{~s}$. The radius of the outer ring is reduced within about two seconds to reach its desired value $R_{o}$ and thereafter it is kept fixed. Averages are performed after about three rotations at $t=180 \mathrm{~s}$ (to get rid of the 
arbitrary initial configuration), and during about one rotation, until $t=239 \mathrm{~s}$. In Fig. 1, a typical snapshot is displayed, where the potential energy of the particles is coded in greyscale so that the stress-chains become visible.

Different global volume fractions $\bar{\nu}$ were examined in the simulations. Here, we present data from three different simulations $\mathrm{A}, \mathrm{B}$, and $\mathrm{C}$ with $\bar{\nu}=0.8084$, 0.8149 and 0.8194 , respectively. For the different simulations the number of large and small particles is $N_{\text {small }}$ : 2511, 2545, 2555 and $N_{\text {large }}$ : 400, 394, 399. For the calculation of the global volume fraction, the small particles glued to the wall are counted with half their volume only, and thus contribute with $\bar{\nu}_{\text {wall }}=$ 0.0047 to $\bar{\nu}$. For more details and material parameters, see (Lätzel et al. 2000b).

\subsection{Volume Fraction}

In Fig. 2, the volume fraction is plotted against the rescaled distance from the inner ring. Starting from an initially uniform volume fraction, a dilated shearzone forms near to the inner wheel as a consequence of the applied shear. This effect is less pronounced for higher initial global densities and, in the outer region of the shear-cell $(\tilde{r}>10)$, the structure of the packing remains frozen, i. e. not much reorganization takes place within the duration of the simulation.

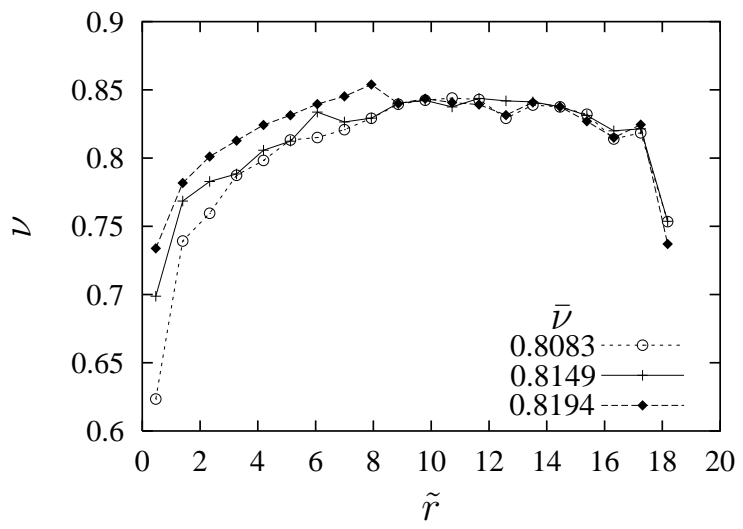

Figure 2: Volume fraction $\nu$, plotted against the dimensionless distance from the origin $\tilde{r}=\left(r-R_{i}\right) / \tilde{d}$, with the typical diameter $\tilde{d}=8 \mathrm{~mm}$, for different initial global densities $\bar{\nu}$

\subsection{Velocity Field}

In Fig. 3, the tangential velocity is plotted against the rescaled distance from the inner ring. The simulation data are fitted by a function $v_{\phi}(r)=v_{0} \exp (-\tilde{r} / s)$ with $v_{o}$ : $0.670,0.756,0.788$ and $s: 1.662,1.584$, 1.191 , thus showing an exponential profile corresponding to the shear band. The shear band, has a width of a few particle diameters, before the velocity $v_{\phi}$ reaches the noise level.

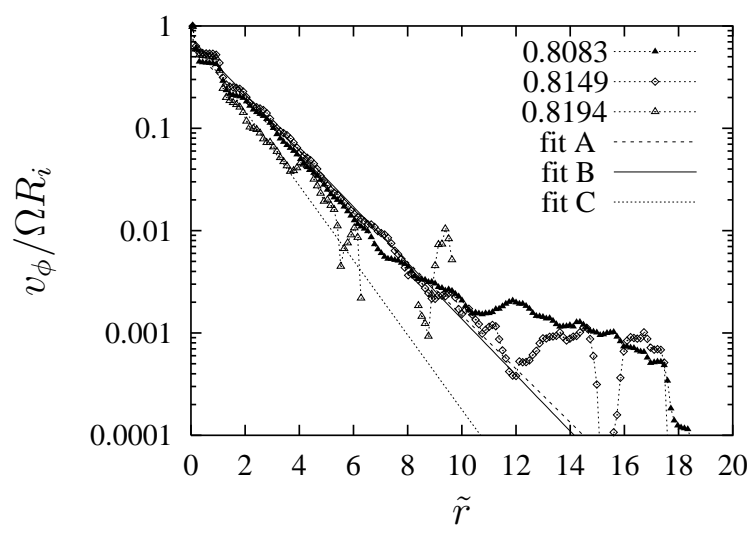

Figure 3: Tangential velocity $v_{\phi}$ normalized by the velocity of the inner wheel $\Omega R_{i}$, plotted against $\tilde{r}$. The lines are the fits to the simulation data for $\tilde{r}=0.25$ to 8.1 , using $v_{\phi}(r)=v_{0} \exp (-\tilde{r} / s)$ with $v_{o}$ : $0.670,0.756,0.788$ and $s: 1.662,1.584,1.191$, for increasing density, as given in the inset, respectively

\subsection{Fabric Tensor}

The trace of the fabric tensor is a measure for the contact number density, whereas the deviator of the fabric is a measure for the anisotropy of the contact network (Lätzel et al. 2000b; Lätzel et al. 2000a), see Fig. 円. In our situation, the contact number density is reduced in the shear zone and the anisotropy, i.e. the deviatoric fraction, is increased in the shear zone, but remains below 20 per-cent.
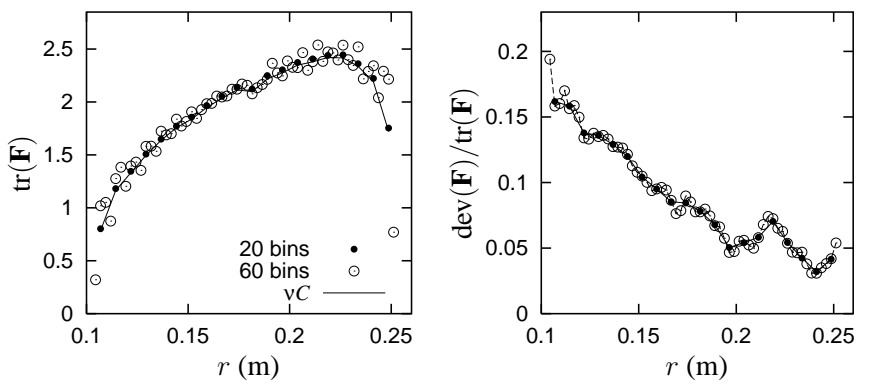

Figure 4: Components of the fabric tensor $\operatorname{tr}(\mathbf{F})$ (isotropic) and $\operatorname{dev}(\mathbf{F}) / \operatorname{tr}(\mathbf{F})$ (anisotropic) for different numbers of binning intervals

\subsection{Stress Tensor}

In Fig. 5, the static stress components are plotted. Here, the diagonal elements are almost constant, whereas the off-diagonal elements decay proportional to $r^{-2}$, as indicated by the lines, in consistency with continuum theoretical equilibrium conditions (Luding et al. 2000). The trace of the stress tensor, 1. e. the volumetric stress, is almost constant over the whole shear-cell besides fluctuations. In contrast, the deviatoric fraction $\sigma_{D} / \sigma_{V}$ decays with increasing distance $r$ from the inner ring, similar to the behavior of the deviatoric fraction of $\mathbf{F}$. 


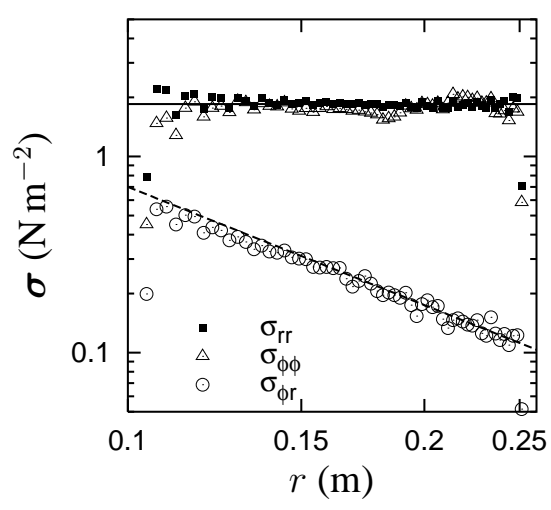

Figure 5: Components of the static stress $\sigma$ plotted against the distance from the center $r$ in log-log representation.

\subsection{Tensor Orientations}

The orientations of the tensors $\mathbf{F}, \boldsymbol{\sigma}$, and $\epsilon$ (data not shown here) are tilted from the radial outwards direction into shear direction (Lätzel et al. 2000b; Luding et al. 2000; Lätzel et al. 2000a). Interestingly, the orientations of the three tensors are different, indicating an anisotropic material. All orientation angles show the same qualitative behavior, however, the fabric is tilted more than the stress which, in turn, is tilted more than the deformation gradient. Thus, the three tensorial quantities examined so far are not co-linear.

\subsection{Angular velocity field}

In Fig. 6 the ( $z$-components of the) macroscopic particle rotations $\omega$, the continuum rotation $\omega_{c}$ and the particle excess-rotation $\omega^{*}=\omega-\omega_{c}$, are displayed. Both the particle- and the continuum-angular velocity decay exponentially with increasing $\tilde{r}$, paralleling the behavior of the velocity $v_{\phi}$. The inset of Fig. 6 shows an oscillation of the excess-rotation near the inner wheel, from one disk layer to the next. This is due to the fact that the disks in adjacent layers are able to roll over each other in the shear zone.

\section{MATERIAL PARAMETERS}

\subsection{Bulk Stiffness}

The material stiffness $\bar{E}=\operatorname{tr}(\boldsymbol{\sigma}) / \operatorname{tr}(\boldsymbol{\epsilon})$ is here defined as the ratio of the volumetric parts of stress and elastic deformation gradient. In a mean field estimate it is found to be proportional to the "microscopic" contact stiffness $k_{n}^{\prime}$ and the trace of the fabric tensor: $\bar{E} \propto\left(k_{n}^{\prime} / 2 \pi\right) \operatorname{tr}(\mathbf{F})$. In Fig. \ the rescaled stiffness of the granular material is plotted against the trace of the fabric. Note that all data collapse almost on a line, but the mean-field value underestimates the simulation data by some per-cent. The few data points which deviate most are close to the boundaries. The deviation from the mean field prediction (solid line in Fig. (7) seems to disappear in the absence of shear.

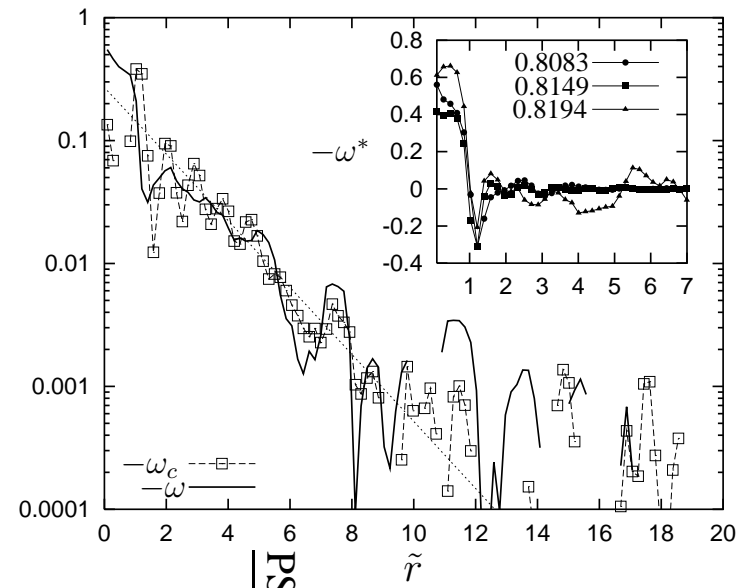

Figure 6: Angular velocities $\omega$ (solid line) and $\omega_{c}$ (symbols) of the particles and the continuum, plotted against the scaled radial distance (from simulation $\mathrm{B}$ ). The dotted line is $\omega_{c}$ as obtained from the fit to $v_{\phi}$, see subsection 3.2. In the inset, the excess-spin is displayed for all simulations

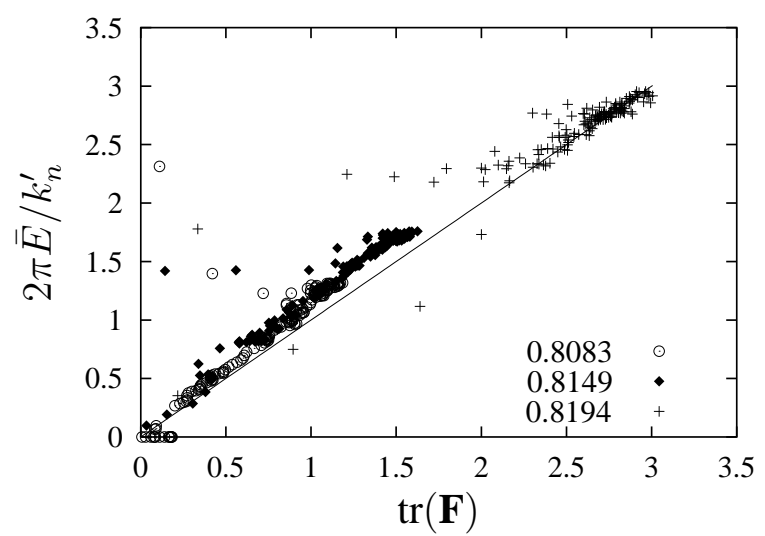

Figure 7: Scaled granular bulk stiffness $2 \pi \bar{E} / k_{n}^{\prime}$, plotted against $\operatorname{tr}(\mathbf{F})$. Every point corresponds to one of 150 rings dividing the system

\subsection{Shear Stiffness}

The typical shear stiffness of the material is defined as the ratio of the deviatoric parts of stress and elastic deformation gradient $G=\operatorname{dev}(\boldsymbol{\sigma}) / \operatorname{dev}(\boldsymbol{\epsilon})$ and scales in a crude mean field approximation as $G \propto\left(k_{n}^{\prime} / \pi\right) \operatorname{tr}(\mathbf{F})$. In Fig. 8 the ratio of the deviatoric parts of stress and strain is plotted against the trace of the fabric. Like the bulk stiffness, both quantities are proportional, at least for points near or within the dilute shear band. In the denser outer part of the shearcell, the particles are strongly inter-locked and thus resist much more against shear, so that $G$ diverges. The critical contact number density grows with increasing global density, i. e. with increasing stress.

\subsection{Torque Resistance}

Since we are interested in the role the rotational degree of freedom plays for the constitutive equations, 


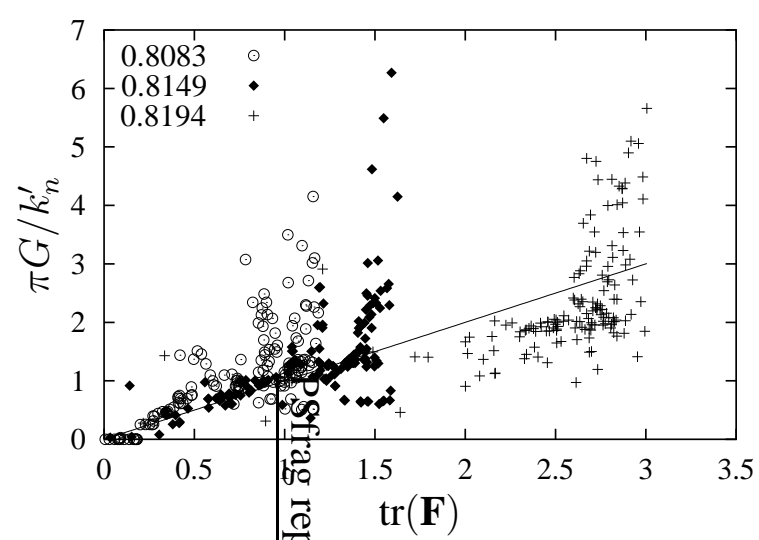

Figure 8: Scaled granular shear resistance $\pi G / k_{n}^{\prime}=$ $\operatorname{dev}(\boldsymbol{\sigma}) / \operatorname{dev}(\boldsymbol{\epsilon})$ plotted against $\operatorname{tr}(\mathbf{F})$ from all simulations. The line indicates the identity curve

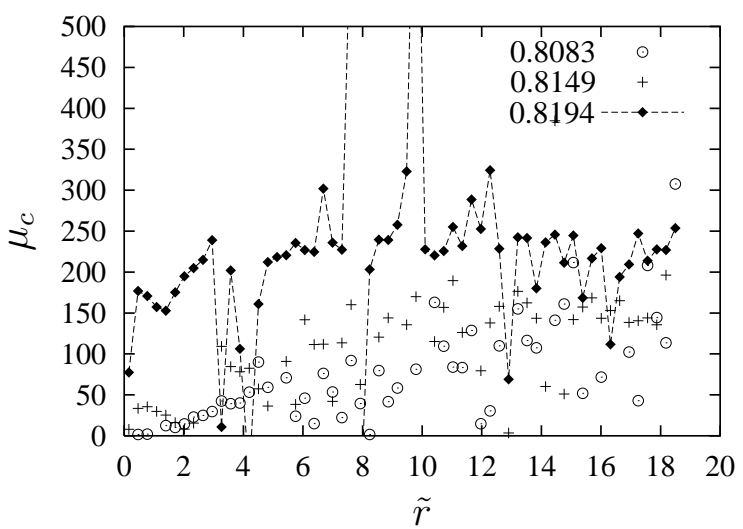

Figure 9: Torque resistance plotted against $\tilde{r}$

we define a new material parameter, the "torque resistance" $\mu_{c}$, as the ratio of the magnitudes of the couple stress and the curvature components. This quantity describes how strongly the material resists against applied torques. In Fig. 9, $\mu_{c}$ is plotted against the scaled distance from the inner ring. In the dilute regions near to the shear zone, where the particles are able to rotate more easily, $\mu_{c}$ is smaller than in the denser regions, where the particles are interlocked and thus frustrated. This is consistent with the results for increasing global densities, i. e. the torque resistance increases with density. Note that the strongest fluctuations are due to the division by small values and have no physical relevance in our interpretation.

\section{SUMMARY AND CONCLUSION}

A micro-macro averaging procedure was presented and specific boundary conditions were chosen to allow for averaging over large volumes and long times. A shear band is obtained, localized close to the inner, rotating cylindrical wall. The local configurations changed rather rapidly in the shear band, whereas the system is almost frozen in the outer part.

The averaging strategy used assumes the quantities to be homogeneously smeared out over the whole particle which is cut in slices by the averaging volumes. The material density, i. e. the volume fraction, the velocity field, the fabric tensor, the stress tensor and the elastic, reversible deformation gradient were obtained from DEM simulations. The volumetric stress is constant in radial direction while its deviatoric fraction and also the mean velocity gradient decay with increasing distance from the inner wall. The ratio of the volumetric parts of stress and strain gives the material stiffness of the granulate, which is small in the shear band, due to dilation, and larger outside.

In the shear-cell, large deviators, i. e. anisotropy, of all tensorial quantities is evidenced. All tensors are tilted from the radial direction. The system organizes itself such that more contacts are created to act against the shear and also the shear resistance increases with the contact density. An essential result is that the macroscopic tensors are not co-linear, i.e. their orientations are different. Thus, the material cannot be described by a simple elastic model involving only the two Lamé constants (or bulk modulus and Poisson's ratio).

Finally, the particles' angular velocity was averaged in analogy to the particle velocity. Subtraction of the continuum rotation from the particle rotation leads to the excess-eigen rotation of the particles with respect to the mean rotation, in the spirit of a micropolar or Cosserat continuum theory. In analogy to the stress and elastic deformation gradient, we defined couple stress and curvature. The quotient of the respective non-zero components gives a "torqueresistance" which increases with increasing local density and stress.

Current research concerns the application of the averaging formalism to other boundary conditions, the use of more realistic particle interaction models, and the measurement of other macroscopic quantities not discussed here.

\section{ACKNOWLEDGEMENTS}

The author thanks M. Lätzel for data and discussions and acknowledges financial support by the Deutsche Forschungsgemeinschaft (DFG).

\section{REFERENCES}

Bathurst, R. J. and L. Rothenburg (1988). Micromechanical aspects of isotropic granular assemblies with linear contact interactions. $J$. Appl. Mech. 55, 17-23.

Behringer, R. P. and J. T. Jenkins (Eds.) (1997). Powders \& Grains 97, Rotterdam. Balkema.

Calvetti, F., G. Combe, and J. Lanier (1997). Experimental micromechanical analysis of a $2 \mathrm{~d}$ granular material: relation between structure 
evolution and loading path. Mech. Coh. Fric. Mat. 2, 121-163.

Cambou, B., F. Dubujet, F. Emeriault, and F. Sidoroff (1995). Homogenization for granular materials. Eur. J. Mech. A/Solids 14(2), 255-276.

Cundall, P. A. and O. D. L. Strack (1979). A discrete numerical model for granular assemblies. Géotechnique 29(1), 47-65.

Dedecker, F., M. Chaze, P. Dubujet, and B. Cambou (2000). Specific features of strain in granular materials. Mech. Coh.-Fric. Mat. 5(3), 174193.

Emeriault, F. and C. S. Chang (1997). Interparticle forces and displacements in granular materials. Computers and Geotechnics 20(3/4), 223-244.

Falcon, E., C. Laroche, S. Fauve, and C. Coste (1998). Behavior of one inelastic ball bouncing repeatedly off the ground. Eur. Phys. J. B 3, 45-57.

Foerster, S. F., M. Y. Louge, H. Chang, and K. Allia (1994). Measurements of the collision properties of small spheres. Phys. Fluids 6(3), 1108-1115.

Herrmann, H. J., J.-P. Hovi, and S. Luding (Eds.) (1998). Physics of dry granular media - NATO ASI Series E 350, Dordrecht. Kluwer Academic Publishers.

Howell, D. and R. P. Behringer (1999). Fluctuations in a $2 \mathrm{~d}$ granular Couette experiment: A critical transition. Phys. Rev. Lett. 82, 5241.

Howell, D. W., R. P. Behringer, and C. T. Veje (1999). Fluctuations in granular media. Chaos 9(3), 559-572.

Kruyt, N. P. and L. Rothenburg (1996). Micromechanical definition of strain tensor for granular materials. ASME Journal of Applied Mechanics 118, 706-711.

Labous, L., A. D. Rosato, and R. Dave (1997). Measurements of collision properties of spheres using high-speed video analysis. Phys. Rev. E 56, 5715.

Lätzel, M., S. Luding, and H. J. Herrmann (2000a). From discontinuous models towards a continuum description. In P. A. Vermeer et al. (Eds.), Continuous and Discontinuous Modelling of Cohesive Frictional Materials, Springer, Berlin, pp. 215-230.

Lätzel, M., S. Luding, and H. J. Herrmann (2000b). Macroscopic material properties from quasi-static, microscopic simulations of a twodimensional shear-cell. Granular Matter 2(3), 123-135. cond-mat/0003180.
Liao, C.-L., T.-P. Chang, D.-H. Young, and C. S. Chang (1997). Stress-strain relationship for granular materials based on the hypothesis of best fit. Int. J. Solids \& Structures 34, 40874100.

Luding, S. (1998). Collisions \& contacts between two particles. In H. J. Herrmann, J.-P. Hovi, and S. Luding (Eds.), Physics of dry granular media - NATO ASI Series E350, Dordrecht, pp. 285-304. Kluwer Academic Publishers.

Luding, S., M. Lätzel, W. Volk, S. Diebels, and H. J. Herrmann (2000). From discrete element simulations to a continuum model. Comp. Meth. Appl. Mech. Engng.. in press.

Madadi, M., O. Tsoungui, M. Lätzel, and S. Luding (2001). On the fabric tensor of static, polydisperse granular materials. in preparation.

Mehrabadi, M. M., S. Nemat-Nasser, H. M. Shodja, and G. Subhash (1988). Some basic theoretical and experimental results on micromechanics of granular flow. In Micromechanics of granular media, Amsterdam. Elsevier.

Rothenburg, L. and A. P. S. Selvadurai (1981). A micromechanical definition of the Cauchy stress tensor for particulate media. In A. P. S. Selvadurai (Ed.), Mechanics of Structured Media, pp. 469-486. Elsevier, Amsterdam.

Satake, M. and J. T. Jenkins (1988). Micromechanics of granular materials. Amsterdam: Elsevier.

Veje, C. T., D. W. Howell, and R. P. Behringer (1999). Kinematics of a 2D granular Couette experiment. Phys. Rev. E 59, 739.

Vermeer, P. A., S. Diebels, W. Ehlers, H. J. Herrmann, S. Luding, and E. Ramm (Eds.) (2000). Continuous and Discontinuous Modelling of Cohesive Frictional Materials, Berlin. Springer. Lecture Notes on Physics 658.

Zervos, A., I. Vardoulakis, M. Jean, and P. Lerat (2000). Numerical investigation of granular interfaces kinematics. Mech. Coh.-Fric. Mat. 5, 305-324. 